\title{
Enhancing Model Robustness and Fairness with Causality: A Regularization Approach
}

\author{
Zhao Wang \\ Kai Shu \\ Aron Culotta \\ University of Chicago Illinois Institute of Technology Tulane University \\ zwang13@uchicago.edu kshulit.edu aculottadtulane.edu
}

\begin{abstract}
Recent work has raised concerns on the risk of spurious correlations and unintended biases in statistical machine learning models that threaten model robustness and fairness. In this paper, we propose a simple and intuitive regularization approach to integrate causal knowledge during model training and build a robust and fair model by emphasizing causal features and de-emphasizing spurious features. Specifically, we first manually identify causal and spurious features with principles inspired from the counterfactual framework of causal inference. Then, we propose a regularization approach to penalize causal and spurious features separately. By adjusting the strength of the penalty for each type of feature, we build a predictive model that relies more on causal features and less on non-causal features. We conduct experiments to evaluate model robustness and fairness on three datasets with multiple metrics. Empirical results show that the new models built with causal awareness significantly improve model robustness with respect to counterfactual texts and model fairness with respect to sensitive attributes.
\end{abstract}

\section{Introduction}

Modern machine learning models are becoming increasingly successful and are widely used in high-stake applications such as filtering loan applicants (Hassani, 2020), determining school admissions (Alvero et al., 2020), and medical diagnosis (Ahuja, 2019), etc. However, a big challenge for statistical machine learning is that the models are data-driven and usually built on statistical correlations that are sometimes spurious. For example, a sentiment classifier trained on IMDB movie reviews predicts "the film directed by Spielberg is incredibly interesting" as positive. While the prediction is correct, the model takes "Spielberg" as a highly predictive feature, that is learned to be strongly correlated with positive sentiment during model training. This is a spurious correlation

\author{
the film directed by Spielberg is incredibly interesting . \\ $0.712 \quad 0.576$ \\ $0.290 \quad 0.874$
}

Table 1: The motivating example: the traditional classifier (1st row) learns spurious correlation between Spielberg and positive sentiment and assigns a large weight for the spurious feature. Our regularization approach (2nd row) encourages the model to assign a smaller weight for the spurious feature and a larger weight for the causal feature (interesting).

since an annotator won't label a review as positive just because it mentions "Spielberg". A classifier built on this spurious correlation might perform well when the testing data has the same distribution as training data (i.e., positive reviews talking about "Spielberg"), however, it is very likely to fail when there's a distribution shift in testing data (i.e., negative reviews talking about "Spielberg"). For another example, a classifier trained on school admission data learns the "gender" attribute to be predictive of admission status. The classifier learns this correlation because the "gender" attribute happens to correlate with admission in the training data that might contain discrimination and societal bias. Such a biased classifier will raise issues of discrimination when deployed in the real world. In the above examples, the spurious correlations are built in the model because those features happen to correlate with a specific class in the training data, but model designers do not want such features to carry predictive power in the model because that will make the model suffer from robustness (e.g., fail on different distributions), fairness (e.g., bias towards specific groups), and trustworthiness (e.g., not convincing when explaining model performance).

Existing works have investigated multiple ways to deal with spurious correlations and improve model robustness and fairness, such as feature selection (Paul, 2017; Wang and Culotta, 2020), data augmentation (Kaushik et al., 2020; Wang 
and Culotta, 2021), instance re-weighting (Zhang et al., 2020), counterfactual logit pairing (Garg et al., 2019), control for confounders (Landeiro and Culotta, 2018), apply constraints to design fair and robust objective functions (Dwork et al., 2012; Zhao et al., 2021). Recent works also explore causal inference for robust and fair models, such as leveraging human commonsense of causal reasoning (Srivastava et al., 2020), adapting text embeddings for causal inference (Veitch et al., 2020), and providing causal views on robustness of neural networks (Zhang and Li, 2020). While effective, most works only deal with either fairness or robustness, some works require significant human efforts, some have trade-offs between model robustness and overall performance on test set, and some are complex to deploy in real-world applications.

In this paper, we propose a simple and intuitive regularization approach to integrate causal knowledge in model training. We assign large regularization penalties on spurious features and small penalties on causal features (i.e., features that cause a sample to get a label). By doing so, we encourage the model to pay less attention to spurious features and more to causal features. Table 1 demonstrates a motivating example, where a traditional sentiment classifier (such as Logistic Regression) assigns a large weight for the spurious feature "Spielberg $(0.874)$ " and a small weight for the causal feature "interesting (0.576)". The new classifier built with our regularization approach decreases the weight of the spurious feature and increases the weight of the causal feature.

Specifically, we first manually identify causal and spurious features based on criteria motivated by the counterfactual framework of causal inference. Then, we incorporate regularization components to add different penalties for different types of features. By adjusting the strength of the penalty for each type of feature and optimizing the customized loss function, we build a model that assigns larger weights to causal features and smaller weights to non-causal features. Finally, we carry out experiments to test whether our approach improves model robustness and fairness. For model robustness, we experiment with IMDB movie reviews and Kindle book reviews text data. We evaluate the model performance on both the standard test set as well as a counterfactual test set. Results show that our models significantly improves robustness on the counterfactual test set compared to the traditional models (e.g., 12\% increase in accuracy for the best model). For model fairness, we experiment with the law school admission dataset and evaluate with two metrics (i.e, equal opportunity and demographic parity). Our models show more fair performance than the traditional model (e.g., females and males almost get equal opportunity and demographic parity in the best model). With this framing, the main contributions of this paper are as follows:

Method: We propose a simple and novel regularization approach to integrate causality during model training. By constructing and optimizing the customized loss function that adjusts the penalty for causal and spurious features, we build predictive models that pay more attention to causal features and less to non-causal features.

Evaluation: We evaluate our proposed models on both robustness (with high-dimensional text data) and fairness (with low-dimensional tabular data). Our models outperform multiple baselines in terms of robustness to counterfactual texts and fairness to sensitive attributes, without sacrificing model performance on test set.

\section{Related Work}

As the main causes for fairness and robustness issues in ML, biases and spurious correlations can be introduced from multiple sources such as culture, data, and algorithm perspectives. Examples include societal discrimination and bias (Mehrabi et al., 2021; Yapo and Weiss, 2018), data imbalance, sampling bias, data poisoning (Zhao et al., 2018; Kiritchenko and Mohammad, 2018; Chen et al., 2017); covariate shift and confounders (Landeiro and Culotta, 2018), over-parameterization (Sagawa et al., 2020b,a).

Fairness in ML can be summarized as individual fairness (Lahoti et al., 2019), group fairness (Hardt et al., 2016), and max-min fairness (Lahoti et al., 2020). Related research typically falls into three categories based on the stage at which fairness is applied: (1) pre-processing: modify training data and revise sensitive attributes (Kamiran and Calders, 2011), generate balanced data, increase data diversity (Xu et al., 2018), instance reweighting (Zhang et al., 2020); (2) in-processing: apply fairness constraints (regularizations) to design objective functions (Dwork et al., 2012; Zhao et al., 2021), adversarially learn fair representations (Beutel et al., 2017); (3) post-processing: use 
active learning to collect feedbacks and correct predictions (Zaidan et al., 2007). Here we focus on group fairness with respect to sensitive attributes.

Robustness in ML-based NLP models: Ribeiro et al. (2020) propose CheckList to identify critical model failures with diverse types of test cases; Garg et al. (2019) introduce hard ablation, blindness, and counterfactual logit pairing to improve counterfactual token fairness. Kaushik et al. (2020) do counterfactual data augmentmentation to improve model robustness. Lu et al. (2020) create gender-balanced dataset to learn embeddings that mitigate gender stereotypes. Others explore robust optimization, adversarial training, and domain adaptation methods to improve model robustness (Namkoong and Duchi, 2016; Beutel et al., 2017; Ben-David et al., 2006).

Recent research draws connections between robustness and causal inference in text. Keith et al. (2020) and Wood-Doughty et al. (2018) provide detailed overviews. The works closely related to this paper include: achieving robustness by leveraging human commonsense and counterfactual reasoning (Srivastava et al., 2020), adopting active learning and feedback mechanism to highlight rationales (Zaidan et al., 2007), automatically generating counterfactuals with causal words (Wang and Culotta, 2021), applying causal inference for feature selection in text classification (Paul, 2017; Wang and Culotta, 2020), controlling for confounders (Landeiro and Culotta, 2018). Additional works integrate causal inference in text representation and deep learning, such as causal embedding (Veitch et al., 2020) and causal view on robustness of neural networks (Zhang and Li, 2020).

Our proposed approach is inherently different from the aforementioned approaches: (1) We propose a general regularization approach to achieve robustness and fairness with causality. While previous works have shown the ability to improve model fairness and robustness, most of them only deal with either fairness (to sensitive attributes) or robustness (to counterfactual examples); (2) Our proposed regularization approach is effective (with high prediction performance), efficient (requires little human annotation), and explainable (larger weights for causal features).

\section{Problem Definition}

Our primary task is given input $x$, where $x$ is represented by a sequence of features $\left[x_{1}, x_{2}, \ldots, x_{n}\right]$, to predict an outcome $y$. We consider the case of a classifier $f$ parameterized by $w$ that produces a prediction $\hat{y}=f_{w}(x)$. The parameters $w$ are learned during model training by optimizing (minimizing) the loss denoted by the error between $y$ and $\hat{y}$.

To clearly illustrate the problem addressed in this paper, we first consider the simple approach of logistic regression classifier $f_{w}(x)=\frac{1}{1+e^{-\langle x, w\rangle}}$ (\$6.2 discusses applying other complex models). We estimate the parameters $w$ on labeled data $\mathcal{X}$ and then examine the (partial) correlations between features and labels by model coefficients (i.e., feature weights).

The goal is to train a model that performs well on test set and generalizes well on data from different distributions, so that the model maintains robustness to counterfactual data as well as fairness to sensitive attributes (e.g., gender, race).

\subsection{Robustness to Counterfactuals}

We measure model robustness on counterfactual samples. If a model is built on spurious correlations, its performance might not drop on a test set that has the same distribution as the training set, but the performance will drop sharply on the counterfactual set, which is created by editing samples from the test set towards a counterfactual label.

Taking text classification as an example, for a piece of text $T$, the corresponding counterfactual sample is created by editing $T$ with minimum changes towards a counterfactual label (Kaushik et al., 2020). For example, for the positive review "the film directed by Spielberg is incredibly interesting", the counterfactual negative review is "the film directed by Spielberg is incredibly boring". If the classifier is built on spurious correlations such as the correlation between "Spielberg" and positive sentiment, it is very likely to make wrong predictions for the counterfactual sentences (e.g., negative reviews talking about "Spielberg"), which makes the classifier suffer from robustness on counterfactual data. However, if the classifier is built on causal associations such as the association between "interesting" and positive sentiment or "boring" and negative sentiment, the model will make right predictions for the counterfactual sentences and for datasets that have different distributions.

\subsection{Fairness to Sensitive Attributes}

Studies have shown that when there are patterns of previous discrimination and societal bias in the 
training data, the trained model is very likely to inherit the bias (Mehrabi et al., 2021; Yapo and Weiss, 2018). In this paper, we study group fairness with respect to sensitive attributes (e.g., gender, race). The goal is to train a model that ensures members of protected groups in the population (e.g., based on sensitive demographic attributes like gender or race) receive "fair share of beneficial outcomes" during model prediction (Hardt et al., 2016).

Taking the law school admission data as an example, we want to train a classifier that is fair to populations of different identity groups defined by sensitive attributes (e.g., gender, race). If the classifier is built on features such as test scores for the admission decision, the model should make fair predictions for applicants from different identity groups. However, if the classifier is built on biased correlations such as using "gender" as a predictive feature for the admission decision, it could potentially harm end-users who identify with those groups and cause ethical concerns.

In this work, we study group fairness with the low-dimensional law school admission data and it's possible to extend the proposed approach to high-dimensional text data with additional text processing. For example, in the task of toxicity prediction of online comments, we can first categorize the comments into different identity groups according to the sensitive attributes they describe (e.g., race, sexual orientation), and then improve classifier fairness with respect to the protected groups using our regularization approach. One big difference between studying fairness with low-dimensional tabular data (e.g., law school admission data) and highdimensional text data is that for low-dimensional data, we directly identify the sensitive attributes as spurious features, but for text data, we need to identify keywords that are descriptive of sensitive attributes as spurious features (e.g., "black" and "white" are reflective of race, "gay" and "straight" are reflective of sexual orientation). We will take this as our future work.

\section{Methods}

To build a model that is robust to counterfactuals and fair to sensitive attributes, we need to deal with issues of spurious correlations and biases. Our solution is a two-stage process. We first manually identify causal and spurious features based on the criteria motivated by the counterfactual framework of causal inference. We then propose a regular- ization approach to build a loss function that adds small penalties for causal features and large penalties for spurious features. By doing so, the model is encouraged to rely more on meaningful causal associations and less on spurious correlations.

\subsection{Annotate Causal and Spurious Features}

Consider an instance $x$ represented by a sequence of features $\left[x_{1}, x_{2}, \ldots, x_{n}\right]$ and having the label $y$. We identify causal and spurious features by using the counterfactual framework of causal inference (Winship and Morgan, 1999): if feature $x_{i}$ were replaced with some other feature $x_{j}$, how likely is it that the label $y$ would change? In this paper, we consider short texts (e.g., single sentences) and low-dimensional tabular data as the unit of analysis, and the following criteria is proposed for this type of data.

A causal feature is a feature $x_{i}$ that causes the sample $x$ to receive the label $y$. All else being equal, one would expect $x_{i}$ to be a determining factor in assigning $y$ to sample $x$. On the other hand, a spurious feature is a feature $x_{i}$ that correlates with the target class $y$ in a specific dataset, but replacing it with another feature $x_{j}$ would be unlikely to change the instance label.

Taking a movie review sentence as an example, "the film directed by Spielberg is incredibly interesting" (pos), "interesting" is a causal feature that is primarily responsible for the positive sentiment and replacing it with another word such as "boring" will change the sentiment to be negative. In contrast, "Spielberg" is a spurious feature as it does not convey positive sentiment nor cause a review to be positive, and replacing it with another word such as "Kevin" will not change the sentiment. Although "Spielberg" might correlate with positive sentiment due to its high frequency in positive movie reviews, this statistical correlation does not imply causality.

With the proposed criteria, we manually annotate causal and spurious features in $\$ 5.2$. Table 2 shows examples of those features identified for sentiment classification and school admission tasks.

Prior works have explored methods that automatically identify causal and spurious features. For example, Wu et al. (2021) design Polyjuice as a general-purpose counterfactual generator that allows for control over multiple perturbation types and the generated counterfactuals are application agnostic. Wang and Culotta (2020) propose to fit a 


\begin{tabular}{l|ll}
\hline & Causal & Spurious \\
\hline \multirow{3}{*}{ Sentiment Classification } & interesting & movie \\
& boring & story \\
& wonderful & Spielberg \\
& inspiring & definitely \\
& enjoyed & animated \\
\hline \multirow{2}{*}{ School Admission } & LSAT & Gender \\
& GPA & Race \\
\hline
\end{tabular}

Table 2: Examples of causal and spurious features

classifier for auto-prediction of causal and spurious features using information such as word embeddings and individual treatment effects. Wang and Culotta (2021) propose a closest opposite matching approach for auto-identification of likely causal features. While these methods work well in general, each has its drawbacks. In this paper, we want to focus on the regularization effect and isolate the noises introduced by other procedures, so we manually annotate causal and spurious features as ground truth.

\subsection{Regularization with Causal and Spurious Features}

Our goal is to train a model that performs well on test data (that has the same distribution as training set) as well as maintains robustness to counterfactuals and fairness to sensitive attributes. Prior works (Kaushik et al., 2020; Wang and Culotta, 2021) have demonstrated the effectiveness of augmenting training data with counterfactuals to improve model robustness. Wang and Culotta (2021) conducted empirical analysis to compare the difference between a traditional model and a robust model, finding that the robust model assigns larger weights for causal features and smaller weights for spurious features.

While previous works have explored the effectiveness of adding L2/L1 penalty (regularization) to prevent over-fitting $(\mathrm{Ng}, 2004)$, the penalty is applied on all features. In this paper, we design a customized loss function that adds different penalties for different types of features (e.g, causal, spurious, and the remaining). The intuition is that when tuning a classifier, we want it to pay more attention to causal features (small penalty) and less attention to spurious features (large penalty). Below we consider three sets of features: causal $(C)$, spurious $(S)$, and remain $(R$, i.e., features except for those labeled as causal or spurious).

Let $J$ be the loss function representing the error/difference between the truth and prediction. To add different penalties for causal, spurious, and remaining features separately, we design the loss function as:

$$
\begin{aligned}
\sum_{x \in X} J(f(x), y) & +\frac{\lambda_{c}}{|C|} \sum_{c \in C} w_{c}{ }^{2} \\
& +\frac{\lambda_{s}}{|S|} \sum_{s \in S} w_{s}{ }^{2} \\
& +\frac{\lambda_{r}}{|R|} \sum_{r \in R} w_{r}{ }^{2}
\end{aligned}
$$

where $\lambda_{c}, \lambda_{s}$, and $\lambda_{r}$ refer to the strength of penalty for causal, spurious, and remaining features; $|C|$, $|S|$, and $|R|$ refer to the size of corresponding feature set; $w_{c}, w_{s}$, and $w_{r}$ refer to the weights of features from each set.

There are many variations of this loss function. For example, we can set $\lambda_{c}=0$ and $\lambda_{r}=0$ to only penalize spurious features. We can also set $\lambda_{c}=0$ to only penalize non-causal features (i.e., a combination of spurious and remain features).

The primary advantage of this approach is that it integrates causal knowledge into model training and provides the flexibility to penalize and address the importance of different types of features. By optimizing the proposed loss function, we seek to build classifiers that satisfy two desirable properties: (1) perform well on testing data and (2) pay more attention to causal features and less to spurious/non-causal features, which is the key to make the model robust and fair on data from different distributions.

\section{Experiments}

We conduct experiments with three datasets to evaluate the effectiveness of the proposed method from two aspects ${ }^{1}$ : robustness to counterfactuals (evaluate on high-dimensional text data from IMDB movie reviews and Kindle book reviews) and fairness to sensitive attributes (evaluate on lowdimensional tabular data of law school admission).

\subsection{Data}

IMDB movie reviews: This dataset is collected and published by Kaushik et al. (2020). They first randomly sampled $2.5 \mathrm{~K}$ reviews with balanced class distributions from the original large IMDB movie reviews dataset (Pang and Lee, 2005) and then instruct Amazon Mechanical Turk workers to

\footnotetext{
${ }^{1}$ Code and data available at: https: / / github.com/ tapilab/emnlp-2021-regularization
} 
edit each review with minimum changes towards a counterfactual label. In the final dataset, every review gets a corresponding counterfactual text. We apply the data splitting criteria from the paper and the train/validation/test sets contain 1,707/245/488 samples. The counterfactual test set is created by counterfactually editing samples from the test set.

Kindle book reviews: This dataset ${ }^{2}$ contains book reviews from the Amazon Kindle store (He and McAuley, 2016) and each review is labeled as positive or negative based on its rating (ratings $\{4,5\}$ as positive and ratings $\{1,2\}$ as negative). To reduce noisy samples, we limit the reviews to those contain 5 to 40 words and the final dataset is split into train/validation/test sets containing 7,500/2,500/500 samples. For the 500 test samples, we manually edit each sample with minimum changes towards a counterfactual label (i.e., the same criteria used in (Kaushik et al., 2020) to edit counterfactuals for IMDB reviews above).

Law school admission data: This dataset contains admission data of 25 US law schools over 2005-2007 ${ }^{3}$. Each sample is an admission record for a student and is represented by seven features: LSAT, GPA, resident, admission year, gender, race, URM (under-represented minority), with a binary label indicating the admission status. The dataset is split into train/validation/test sets containing 50\%, $20 \%$, and $30 \%$ samples.

For the IMDB and Kindle datasets, each sample $x$ is a piece of text, represented by a binary, bag-ofwords representation $x=\left[x_{1}, x_{2}, \ldots, x_{v}\right]$, where $v$ is the vocabulary size and $x_{i}$ is a binary value indicating the appearance of a word in the text. Compared with the high-dimensional text data, the law school admission data is low-dimension with each sample represented by seven numerical or categorical features. We process this dataset by first applying one-hot encoding for categorical features and then normalizing numerical features into range 0-1. In the experiments below, we show that our method works well on both high- and lowdimensional data.

\subsection{Causal and Spurious Features}

With the criteria proposed in 4.1, we ask two student annotators to manually label causal and spurious features from each dataset. Specifically,

\footnotetext{
${ }^{2}$ https: / / jmcauley.ucsd. edu/data / amazon/

${ }^{3}$ http: //www. seaphe.org/databases.php
}

\begin{tabular}{l|lll}
\hline & IMDB & Kindle & Admission \\
\hline \#Instances & $2.5 \mathrm{k}$ & $10.5 \mathrm{k}$ & $65.3 \mathrm{k}$ \\
\#Features & 2,388 & 2,041 & 7 \\
\#Causal(top) & 65 & 76 & 2 \\
\#Spurious(top) & 166 & 118 & 1 \\
\#Causal(vocabulary) & 362 & 293 & 2 \\
\hline
\end{tabular}

Table 3: Data summary

for each dataset, we first train an initial classifier and extract top coefficient features (e.g. coefficient magnitude $>1$ ). For example, for logistic regression model, we would extract features with high magnitude coefficients. For more complex models, other transparency algorithms may be used (Martens and Provost, 2014).

For the IMDB and Kindle datasets, the annotators identified two sets of causal and spurious features: the first set is annotated from the top coefficient features and the second set is annotated from the whole vocabulary (the first set is a subset of the second set). While there is some subjectivity during annotation, we did a round of training to resolve disagreements prior to annotation and the final agreement was generally high for the experimental datasets (on average 96\% raw agreement by fraction of labels that agree).

For the law school admission dataset, there are only seven features and the annotators directly identify "LSAT" and "GPA" as causal features and "gender" as a spurious feature (i.e., sensitive attribute). There are other spurious features such as "race" and "URM", but for this paper, we only focus on model fairness with respect to gender (to have a direct comparison with previous work), so we identify "gender" as a spurious feature and take other potential spurious features as "remaining" features.

Table 3 summarizes the number of identified causal and spurious features for each dataset.

\subsection{Experimental Settings}

Among the numerous works focused on improving model robustness to counterfactuals, the ones most closely related to ours include: augmenting training data with counterfactuals to train a robust classifier (Kaushik et al., 2020; Wang and Culotta, 2021); a logit pairing strategy to penalize the norm of difference in logits of training examples and their counterfactuals (Garg et al., 2019); causal inference for feature selection (Paul, 2017); a feature selection strategy to remove spurious features and improve model robustness with respect to the worst-case accuracy (Wang and Culotta, 2020). 


\begin{tabular}{l|lll|lll}
\hline & \multicolumn{3}{|c|}{ IMDB } & \multicolumn{3}{c}{ Kindle } \\
Method & & Test & CTF & & Test & CTF \\
\hline Feature selection & & $0.835 \pm 0.005$ & $0.633 \pm 0.012$ & & $0.850 \pm 0.002$ & $0.510 \pm 0.017$ \\
Data augmentation & & 0.818 & 0.869 & & 0.752 & 0.720 \\
L2 with BOW & & $0.845 \pm 0.005$ & $0.567 \pm 0.009$ & & $0.853 \pm 0.027$ & $0.412 \pm 0.048$ \\
L2 with Glove & & $0.824 \pm 0.002$ & $0.619 \pm 0.006$ & & $0.820 \pm 0.004$ & $0.553 \pm 0.027$ \\
\hline Regularization $($ top $)$ & $\lambda_{c}, \lambda_{s}, \lambda_{r}$ & & & $\lambda_{c}, \lambda_{s}, \lambda_{r}$ & & \\
\hline causal+spurious & $(0,10,0)$ & $0.836 \pm 0.006$ & $0.632 \pm 0.005$ & $(0,10,0)$ & $0.857 \pm 0.007$ & $0.545 \pm 0.013$ \\
causal+non-causal & $(0,100,100)$ & $0.821 \pm 0.011$ & $0.658 \pm 0.01$ & $(0,10,10)$ & $0.843 \pm 0.003$ & $0.557 \pm 0.003$ \\
causal+spurious+remain & $(0,100,0.1)$ & $0.841 \pm 0.007$ & $0.635 \pm 0.006$ & $(0,100,1)$ & $0.847 \pm 0.003$ & $0.571 \pm 0.008$ \\
\hline Regularization (vocab) & & & & & & \\
causal+spurious & $(0,10,0)$ & $0.838 \pm 0.004$ & $0.634 \pm 0.007$ & $(0,100,0)$ & $0.857 \pm 0.003$ & $0.545 \pm 0.01$ \\
causal+non-causal & $(0,100,100)$ & $0.843 \pm 0.005$ & $0.700 \pm 0.007$ & $(0,10,10)$ & $0.855 \pm 0.005$ & $0.641 \pm 0.006$ \\
causal+spurious+remain & $(0.01,100,100)$ & $0.840 \pm 0.004$ & $0.697 \pm 0.008$ & $(0.001,1,10)$ & $0.848 \pm 0.006$ & $0.636 \pm 0.003$ \\
\hline
\end{tabular}

Table 4: Robustness evaluation for tuned classifiers. Accuracy reported for test and Counterfactual (CTF) data from IMDB and Kindle dataset. Results in 1st section are baseline and state-of-the-art methods, 2nd section shows regularization approach using causal features identified from top coefficient terms and 3rd section shows regularization approach using causal features identified from the whole vocabulary.

We compare our proposed regularization approach with the representative and state-of-the-art prior works:

- Feature Selection (Wang and Culotta, 2020): we explore whether directly removing spurious features is more effective than penalizing spurious features with regularization approach.

- Data Augmentation (Wang and Culotta, 2021): Augmenting original training data with corresponding counterfactual data (either automatically generated or manually edited). This method combines counterfactuals as part of the training data and changes data distribution and data size. It usually requires significant human efforts to generate/edit counterfactuals.

For our proposed regularization approach, we experiment with the LogisticRegression classifier and explore the following settings:

- L2-regularization: as representative of the traditional regularization approach.

- Feature representation for text: we explore Bag-Of-Words (BOW) and pre-trained Glove embedding (Pennington et al., 2014) representations to check whether complex representations help improve robustness.

- The number of causal and spurious features: we experiment with two sets of causal and spurious features: (a) a small set identified from top coefficient features and (b) a full set identified from the whole vocabulary. The goal is to check whether providing more causal features improves model robustness.

Hyper-parameter tuning: we use BinaryCrossEntropy as the basic loss function in all models and implement the regularization approach by customizing the loss function with penalties for causal, spurious, and remaining features as discussed in $\S 4.2$. The penalty strengths (i.e., $\left.\lambda_{c}, \lambda_{s}, \lambda_{r}\right)$ are explored in the range [0, 0.0001, $0.001,0.01,0.1,1,10,100,1000]$ with the constraint that $l_{s} \geq l_{r}>l_{c}$. We use Adam optimizer and set the initial learning rate to 0.001 , and adjust the learning rate according to different $\lambda$ values to avoid overshooting. We use early stopping criteria on validation data to determine the epoch to stop tuning (i.e., stop tuning if the validation loss hasn't decreased for 10 epochs). Among all the tuned models, we select the best model based on its performance on the counterfactual validation data.

After selecting the best model, we evaluate its performance on the test set and counterfactual set. We repeat the training process 10 times with different random seeds and report the model performance and corresponding hyper-parameter settings in Table 4. All experiments are conducted on a 64-bit machine with Nvidia GPU (Tesla V100, 1246MHz, 16 GB memory).

\subsection{Robustness Evaluation}

We evaluate model robustness on the counterfactual texts from IMDB and Kindle datasets. We train different models by optimizing the customized loss functions suggested in $\$ 4.2$ and evaluate model performance on the test set and counterfactual set. The best model parameter setting and performance is reported in Table 4.

Results show that: (1) our proposed regularization approach outperforms feature selection on the counterfactual test set without sacrificing perfor- 
mance on the test set; data augmentation outperforms our regularization approach on the counterfactual set but sacrifices performance on the test set, which is expected because data augmentation approach increased training data size and changed training data distribution. And data augmentation usually requires more human efforts than our regularization approach. (2) separately penalizing causal, spurious, and remaining features is more effective than L2 regularization in terms of model robustness on counterfactual set. The best performance is achieved by assigning no/small penalty for causal features and large penalties for spurious and remaining features; (3) the more causal features provided, the better robustness on counterfactual set. For IMDB, the best model gives $9 \%$ improvement with top causal features and $13.3 \%$ with all causal features. For Kindle, the best model gives $15.9 \%$ improvement with top causal features and $22.9 \%$ with all causal features; (4) complex text representation (e.g., embedding) has limited improvements on model robustness.

Note that the results and parameter settings in Table 4 are based on the exploration of penalty strengths (i.e., $\lambda_{c}, \lambda_{s}, \lambda_{r}$ ) in the range of [0, 0.0001, $0.001,0.01,0.1,1,10,100,1000]$. The best parameter settings might change for different datasets and larger search space. According to our exploration about the effect of hyperparameter $\lambda$ on model performance in $\S 6.1$, we recommend to set $\lambda_{c}<=0.01, \lambda_{s}>=1$, and $\lambda_{r}>=1$ for experiments on new datasets that don't have counterfactual validation set to decide the best hyperparameter setting. As a reference, when we fix $\lambda_{c}=0$, $\lambda_{s}=100$, and $\lambda_{r}=10$ for all datasets, the accuracy is within $97 \%$ of the best found setting.

We also show the percentage of causal features among top-n large coefficient features of each model in Fig 1. Top features are features that are most important and predictive of the classes, and a high percentage of causal features means that the model relies more on causal features and less on others. We can see that models tuned from our proposed loss functions have much higher percentage of causal features compared with the baselines (L2 and feature selection). The model that only considers causal and spurious features has a relative lower percentage compared with the other two models that consider causal, spurious, and remaining features. Taking the model that separately penalizes causal and non-causal features for an ex-
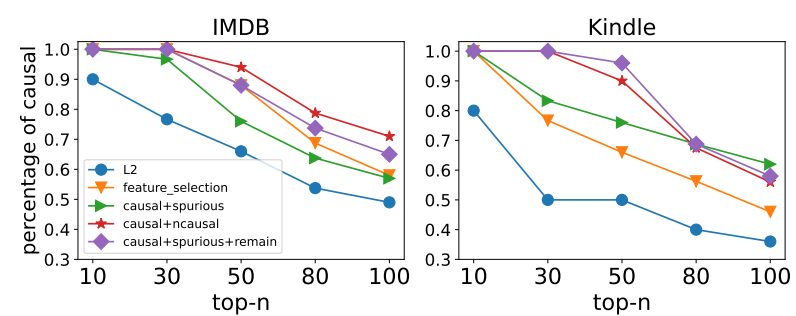

Figure 1: Percentage of causal features among the top$\mathrm{n}$ features of each model. The three models tuned with penalties for causal, spurious, and remain features refer to the models shown in the last three lines in Table 4 (best viewed with color).

ample (IMDB), all features in the top-10 and top-30 are causal features, and about $93 \%$ of top-50 and $70 \%$ of top- 100 are causal features. The difference is even bigger for the Kindle dataset. However, for the baseline models, the percentage is much lower. The high percentage of causal features indicates that our regularization approach successfully makes the model rely more on causal features.

\subsection{Fairness Evaluation}

We experiment with law school admission dataset and ask the question:"does a model trained on this dataset makes fair admission predictions for female and male students?" We apply the same baseline and training procedure described in $\$ 5.4$ with "LSAT" and "GPA" as causal features and "gender" as spurious feature (i.e., sensitive attribute). Following existing work on fairness evaluation (Zhao et al., 2021), we adopt two widely used evaluation metrics, equal opportunity and demographic parity.

Equal opportunity evaluates whether a classifier gives similar results for different sensitive groups in the positive class (Verma and Rubin, 2018; Hardt et al., 2016; Yan et al., 2020). We calculate equal opportunity difference $\Delta_{E O}$ to measure whether a classifier is fair for gender attribute:

$$
\begin{aligned}
\Delta_{E O} & =\mid \operatorname{Pr}\{\hat{y}=1 \mid S=i, y=1\} \\
& -\operatorname{Pr}\{\hat{y}=1 \mid S=j, y=1\} \mid
\end{aligned}
$$

where $S$ is sensitive attribute, $i$ and $j$ are sensitive attribute values defined by $S$ (e.g, male and female defined by gender), and $y$ is the truth label.

In our experiment with law school admission data, if a model is fair, then the probability of an applicant being predicted to be admitted $(\hat{y}=1)$ should be the same for both male $(S=i)$ and female $(S=j)$ applicants that are actually being admitted $(y=1)$. 


\begin{tabular}{llllll}
\hline Penalty & $\lambda_{c}, \lambda_{s}, \lambda_{r}$ & accuracy & $\mathrm{f} 1$ & $\Delta_{E O}$ & $\Delta_{D P}$ \\
\hline L2 (baseline) & $\mathrm{N} / \mathrm{A}$ & $0.796 \pm 0.001$ & $0.650 \pm 0.012$ & $0.102 \pm 0.003$ & $0.028 \pm 0.001$ \\
\hline causal+spurious & $(0.0001,10,0)$ & $0.795 \pm 0.003$ & $0.654 \pm 0.011$ & $0.023 \pm 0.005$ & $0.007 \pm 0.001$ \\
causal + non-causal & $(0.0001,0.001,0.001)$ & $0.796 \pm 0.001$ & $0.655 \pm 0.012$ & $0.100 \pm 0.006$ & $0.027 \pm 0.001$ \\
causal+spurious+remain & $(0.0001,0.1,0.01)$ & $0.794 \pm 0.003$ & $0.646 \pm 0.01$ & $0.028 \pm 0.005$ & $0.004 \pm 0.002$ \\
\hline FairRF (Zhao et al., 2021) & N/A & $0.796 \pm 0.002$ & N/A & $0.023 \pm 0.008$ & $0.007 \pm 0.004$ \\
\hline
\end{tabular}

Table 5: Tuned classifier performance on law school admission test set and fairness evaluation
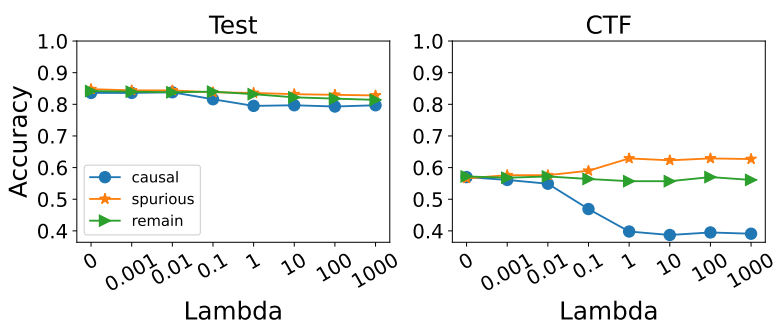

Figure 2: Model performance change with lambda (IMDB) (best viewed with color).

Demographic Parity measures non- discriminative performance of a classifier. We calculate demographic parity difference $\Delta_{D P}$ to check whether a classifier is fair for different sensitive groups:

$$
\begin{aligned}
\Delta_{D P} & =\operatorname{Pr}\{\hat{y}=1 \mid S=i\} \\
& -\operatorname{Pr}\{\hat{y}=1 \mid S=j\}
\end{aligned}
$$

We report the evaluation results from the best model and corresponding model parameters in Table 5. Results show that: (1) the model trained with our regularization approach is more fair with respect to the gender attribute and has comparable performance with previous work using complex models (Zhao et al., 2021); (2) model fairness is achieved by assigning small/no penalties for causal features and large penalties for non-causal features, which is similar as achieving robustness.

\section{Discussion}

\subsection{The Effect of Hyperparameter $\lambda$}

To understand the effect of $\lambda_{c}, \lambda_{s}$, and $\lambda_{r}$ on model performance, we conduct experiments to first initialize $\lambda$ to 0 , and then change the value of each $\lambda$ (Fig 2). We observe that: (1) model performance on test set is relatively stable with different $\lambda$ parameters; (2) model performance on counterfactual set is mainly affected by $\lambda_{c}$. By increasing $\lambda_{c}$, we assign larger penalty on causal features and the model performance drops. In contrast, by increasing $\lambda_{s}$, we assign larger penalty on spurious features and the model performance increases. Penalizing causal features affects model performance more than penalizing spurious features, and penalizing remaining features has the least effect.

\subsection{Extension in Deep Learning Framework}

In the experiments, we use LogisticRegression as our basic classifier because it's intuitive to explain feature weights and penalties. But our main idea is to integrate causality in model training by adding small penalties to causal features and large penalties to non-causal features, and the loss function is not limited to any specific model, so we do think that our approach can be extended to more complex models. For example, possible ways to apply the idea to LSTM classifiers include: (1) assign different attentions for causal, spurious, and remaining features in the input; (2) specify different probabilities to mask out or drop out spurious and causal feature. We leave this part as our future work.

\section{Conclusions}

In this paper, we have introduced the idea of integrating causal knowledge through a regularization approach. We implement this by first identifying causal and spurious features and then assigning different penalties for causal and non-causal features in the loss function. The resulting model assigns larger weights for causal features and smaller weights for spurious features. Experiments have demonstrated that the tuned model is effective to improve robustness and fairness and it works well for both low- and high- dimensional data. The proposed idea is simple to implement, intuitive to explain, has the potential to be easily deployed to other frameworks and various tasks on model robustness and fairness. For future works, we will design automatic methods to identify causal and spurious features and extend the regularization approach to complex deep learning frameworks.

\section{Acknowledgments}

This research was funded in part by the National Science Foundation under grant \#1618244. We would also like to thank the anonymous reviewers for useful feedbacks. 


\section{References}

A. Ahuja. 2019. The impact of artificial intelligence in medicine on the future role of physician. PeerJ, 7.

A.J. Alvero, Noah Arthurs, anthony lising antonio, Benjamin W. Domingue, Ben Gebre-Medhin, Sonia Giebel, and Mitchell L. Stevens. 2020. Ai and holistic review: Informing human reading in college admissions. In Proceedings of the AAAI/ACM Conference on AI, Ethics, and Society, AIES '20, page 200-206, New York, NY, USA. Association for Computing Machinery.

Shai Ben-David, John Blitzer, K. Crammer, and Fernando C Pereira. 2006. Analysis of representations for domain adaptation. In NIPS.

Alex Beutel, Jilin Chen, Zhe Zhao, and Ed H. Chi. 2017. Data decisions and theoretical implications when adversarially learning fair representations. ArXiv, abs/1707.00075.

Xinyun Chen, Chang Liu, Bo Li, Kimberly Lu, and Dawn Song. 2017. Targeted backdoor attacks on deep learning systems using data poisoning. arXiv preprint arXiv:1712.05526.

C. Dwork, Moritz Hardt, T. Pitassi, O. Reingold, and R. Zemel. 2012. Fairness through awareness. ArXiv, abs/1104.3913.

Sahaj Garg, Vincent Perot, Nicole Limtiaco, Ankur Taly, Ed H. Chi, and Alex Beutel. 2019. Counterfactual fairness in text classification through robustness. In Proceedings of the 2019 AAAI/ACM Conference on AI, Ethics, and Society, AIES'19.

Moritz Hardt, EricPrice, and NathanSrebro. 2016. Equality of opportunity in supervised learning. In NIPS.

Bertrand Hassani. 2020. Societal biases reinforcement through machine learning: A credit scoring perspective.

Ruining He and Julian McAuley. 2016. Ups and downs. $W W W^{\prime} 16$.

F. Kamiran and T. Calders. 2011. Data preprocessing techniques for classification without discrimination. Knowledge and Information Systems, 33:1-33.

Divyansh Kaushik, Eduard Hovy, and Zachary Lipton. 2020. Learning the difference that makes a difference with counterfactually-augmented data. In ICLR'20.

Katherine Keith, David Jensen, and Brendan O'Connor. 2020. Text and causal inference: A review of using text to remove confounding from causal estimates. In Proceedings of the 58th Annual Meeting of the Association for Computational Linguistics, pages 5332-5344, Online. ACL.
Svetlana Kiritchenko and Saif Mohammad. 2018. Examining gender and race bias in two hundred sentiment analysis systems. In Proceedings of the Seventh Joint Conference on Lexical and Computational Semantics, New Orleans, Louisiana. ACL.

Preethi Lahoti, Alex Beutel, Jilin Chen, Kang Lee, Flavien Prost, Nithum Thain, Xuezhi Wang, and Ed H. Chi. 2020. Fairness without demographics through adversarially reweighted learning. ArXiv, abs/2006.13114.

Preethi Lahoti, K. Gummadi, and G. Weikum. 2019. Operationalizing individual fairness with pairwise fair representations. Proc. VLDB Endow., 13:506518.

Virgile Landeiro and Aron Culotta. 2018. Robust text classification under confounding shift. Journal of Artificial Intelligence Research, 63:391-419.

Kaiji Lu, Piotr Mardziel, Fangjing Wu, Preetam Amancharla, and Anupam Datta. 2020. Gender bias in neural natural language processing. In Logic, Language, and Security.

David Martens and Foster Provost. 2014. Explaining data-driven document classifications. Mis Quarterly, 38(1):73-100.

Ninareh Mehrabi, Fred Morstatter, Nripsuta Saxena, Kristina Lerman, and Aram Galstyan. 2021. A survey on bias and fairness in machine learning. 54(6).

Hongseok Namkoong and John C. Duchi. 2016. Stochastic gradient methods for distributionally robust optimization with f-divergences. In NIPS.

A. Ng. 2004. Feature selection, 11 vs. 12 regularization, and rotational invariance. Proceedings of the twentyfirst international conference on Machine learning.

Bo Pang and Lillian Lee. 2005. Seeing stars: Exploiting class relationships for sentiment categorization with respect to rating scales. In Proceedings of the 43rd annual meeting on association for computational linguistics, pages 115-124. ACL.

Michael J. Paul. 2017. Feature selection as causal inference: Experiments with text classification. In Proceedings of the 21st Conference on Computational Natural Language Learning (CoNLL 2017).

Jeffrey Pennington, Richard Socher, and Christopher D. Manning. 2014. Glove: Global vectors for word representation. In Empirical Methods in Natural Language Processing (EMNLP), pages 1532-1543.

Marco Túlio Ribeiro, Tongshuang Wu, Carlos Guestrin, and Sameer Singh. 2020. Beyond accuracy: Behavioral testing of nlp models with checklist. In $A C L$.

Shiori Sagawa, Pang Wei Koh, Tatsunori B Hashimoto, and Percy Liang. 2020a. Distributionally robust neural networks for group shifts: On the importance of regularization for worst-case generalization. ICLR. 
Shiori Sagawa, Aditi Raghunathan, Pang Wei Koh, and Percy Liang. 2020b. An investigation of why overparameterization exacerbates spurious correlations. arXiv preprint arXiv:2005.04345.

Megha Srivastava, Tatsunori B. Hashimoto, and Percy Liang. 2020. Robustness to spurious correlations via human annotations. In ICML.

Victor Veitch, Dhanya Sridhar, and David Blei. 2020 Adapting text embeddings for causal inference. In Proceedings of the 36th Conference on Uncertainty in Artificial Intelligence (UAI), volume 124 of Proceedings of Machine Learning Research. PMLR.

Sahil Verma and Julia Rubin. 2018. Fairness definitions explained. In 2018 IEEE/ACM International Workshop on Software Fairness (FairWare).

Zhao Wang and A. Culotta. 2021. Robustness to spurious correlations in text classification via automatically generated counterfactuals. In $A A A I$.

Zhao Wang and Aron Culotta. 2020. Identifying spurious correlations for robust text classification. In Findings of the Association for Computational Linguistics, EMNLP 2020.

Christopher Winship and Stephen L Morgan. 1999. The estimation of causal effects from observational data. Annual review of sociology, 25(1):659-706.

Zach Wood-Doughty, Ilya Shpitser, and Mark Dredze. 2018. Challenges of using text classifiers for causal inference. EMNLP.

Tongshuang Wu, Marco Tulio Ribeiro, Jeffrey Heer, and Daniel S. Weld. 2021. Polyjuice: Generating counterfactuals for explaining, evaluating, and improving models. In Proceedings of the 59th Annual Meeting of the Association for Computational Linguistics. Association for Computational Linguistics.

Depeng Xu, Shuhan Yuan, L. Zhang, and Xintao Wu. 2018. Fairgan: Fairness-aware generative adversarial networks. 2018 IEEE International Conference on Big Data (Big Data), pages 570-575.

Shen Yan, H. Kao, and Emilio Ferrara. 2020. Fair class balancing: Enhancing model fairness without observing sensitive attributes. Proceedings of the 29th ACM International Conference on Information \& Knowledge Management.

Adrienne Yapo and Joseph W. Weiss. 2018. Ethical implications of bias in machine learning. In HICSS.

Omar Zaidan, Jason Eisner, and Christine Piatko. 2007. Using "annotator rationales" to improve machine learning for text categorization. In Human Language Technologies 2007. ACL.

Cheng Zhang and Yingzhen Li. 2020. A causal view on robustness of neural networks.
Guanhua Zhang, Bing Bai, Junqi Zhang, Kun Bai, Conghui Zhu, and T. Zhao. 2020. Demographics should not be the reason of toxicity: Mitigating discrimination in text classifications with instance weighting. In $A C L$.

Jieyu Zhao, Tianlu Wang, Mark Yatskar, Vicente Ordonez, and Kai-Wei Chang. 2018. Gender bias in coreference resolution: Evaluation and debiasing methods. ArXiv.

Tianxiang Zhao, Enyan Dai, Kai Shu, and Suhang Wang. 2021. You can still achieve fairness without sensitive attributes: Exploring biases in nonsensitive features. ArXiv, abs/2104.14537. 